\title{
Different methods for administering $17 \beta$-estradiol to ovariectomized rats result in opposite effects on ischemic brain damage
}

\author{
Jakob O Strom¹, Elvar Theodorsson ${ }^{1 *}$, Lovisa Holm¹, Annette Theodorsson ${ }^{1,2}$
}

\begin{abstract}
Background: Numerous stroke studies have controversially shown estrogens to be either neuroprotective or neurodamaging. The discordant results observed in rat brain ischemia models may be a consequence of discrepancies in estrogen administration modes resulting in plasma concentration profiles far from those intended. To test this hypothesis we reproduced in detail and extended an earlier study from our lab using a different mode of $17 \beta$-estradiol administration; home-made silastic capsules instead of commercial slow-release $17 \beta$-estradiol pellets. Four groups of female rats $(n=12)$ were ovariectomized and administered $17 \beta$-estradiol or placebo via silastic capsules. All animals underwent MCAo fourteen days after ovariectomy and were sacrificed three days later.

Results: In contrast to our earlier results using the commercial pellets, the group receiving $17 \beta$-estradiol during the entire experiment had significantly smaller lesions than the group receiving placebo (mean \pm SEM: $3.85 \pm 0.70 \%$ versus $7.15 \pm 0.27 \%$ of total slice area, respectively; $p=0.015$ ). No significant neuroprotection was found when the $17 \beta$-estradiol was administered only during the two weeks before or the three days immediately after MCAO.

Conclusions: The results indicate that different estrogen treatment regimens result in diametrically different effects on cerebral ischemia. Thus the effects of estrogens on ischemic damage seem to be concentration-related, with a biphasic, or even more complex, dose-response relation. These findings have implications for the design of animal experiments and also have a bearing on the estrogen doses used for peri-menopausal hormone replacement therapy.
\end{abstract}

\section{Background}

During recent years substantial research efforts have been allocated to investigating the potential beneficial effects of estrogens on stroke incidence and mortality. The hypothesis that estrogens may have neuroprotective properties evolved from the observation that premenopausal women have lower risk of stroke than men of the same age have, and that stroke incidence amongst women increases in menopause [1-3]. Estrogens are widely administered as peri-menopausal hormone replacement therapy (HRT), and several large studies on human populations have been devoted to investigating the relationship between HRT and stroke. Of these, some have shown neuroprotection [4,5], while others have cast this effect of estrogens and HRT in doubt

\footnotetext{
* Correspondence: elvar.theodorsson@liu.se

${ }^{1}$ Institution of Clinical and Experimental Medicine/Department of Clinical Chemistry, Linkoping University, Linkoping, Sweden
}

[6-9]. The largest, the Women's Health Initiative (WHI), including more than 16,000 women, was interrupted prematurely because of findings of an increased risk of coronary heart disease, breast cancer and stroke [9].

The fact that the WHI study was discontinued because of HRT-related health risks strongly reduces the possibility of performing further similar studies in human populations concerning HRT and stroke. This currently leaves us with the use of animal models to elucidate the effects of estrogens in stroke. Therefore the importance of animal studies has increased, and even more since they are sine qua non for investigating the detailed biological mechanisms of estrogens. Several studies have been performed on the question of estrogens and ischemic brain damage in animal models, rat being most commonly used. As in the human population studies the results of animal studies have been varying. Although a majority of studies have shown neuroprotective effects of estrogens [10-15], some 
have also shown increased damage after stroke in estrogen-supplemented animals [16-20], among these a study from our laboratory [21]. In a recent review we hypothesized that the divergent results were a consequence of differences in doses of estrogens delivered by different administration modes, commercial pellets being the only method resulting in increased lesions [22]. To test this hypothesis we designed an experiment that exactly mimicked an earlier experiment from our lab, except for the mode of administrating $17 \beta$-estradiol, the most potent of naturally occurring estrogens [21]. Silastic capsules filled with $17 \beta$-estradiol in oil were chosen since they have been shown to produce the most physiologically relevant $17 \beta$-estradiol serum levels, in contrast to the earlier used slow-release pellets from Innovative Research of America (IRA), which have been shown to produce early, very high hormone peaks $[23,24]$. However, the slow-release pellets are available for several doses and release-periods, and our conclusions are obviously limited to the pellets tested. Also, although the $17 \beta$-estradiol levels produced by the silastic capsules can be seen as more physiological, even this hormone profile is artificial because of its lack of natural cyclic variation. Having earlier shown neurodamage in altogether 22 rats receiving supraphysiological doses of $17 \beta$-estradiol [21], we considered it more innovative to study when $17 \beta$-estradiol has its potential effect on cerebral ischemia by adding two groups receiving the hormone only before or only after the MCAo, respectively, rather than to include high-dose estradiol groups by means of slow release pellets or silastic capsules.

\section{Methods}

\section{Animals}

Forty-eight female Sprague Dawley rats were obtained from B\&K Universal (Sollentuna, Sweden). The rats were kept at constant room temperature $\left(21^{\circ} \mathrm{C}\right)$ with 12-h light/dark and sound (soft radio music) cycles for at least one week prior to the experiment. At the start of the experiment the rats were 12-14 weeks of age and weighed $270 \pm 3 \mathrm{~g}$ (mean \pm SEM). The animals were housed 2-4 in each cage, with food (Lactamin, Vadstena, Sweden) and water provided ad libitum. The cages were $20 \mathrm{~cm}$ high and had a floor area of $59.5 \times 38.0 \mathrm{~cm}$. All procedures were conducted in accordance with the National Committee for Animal Research in Sweden and Principles of Laboratory Animal Care (NIH publication no. 86-23, revised 1985). The study was approved by the Local Ethics Committee for Animal Care and Use at Linköping University. The animals used in this experiment were also included in a study of neuropeptide levels in the brain.

\section{Ovariectomy and $17 \beta$-estradiol administration Experimental groups}

Before experimental procedures, the rats were randomly allocated into four groups ( $\mathrm{n}=12$ per group). All animals were anesthetized with $1.2-1.4 \%$ (4.2\% for induction) isoflurane (Forene, Abbott, Scandinavia AB, Kista, Sweden) in an oxygen/nitrous oxide mixture $(30 \% / 70 \%)$ and ovariectomized via the dorsal route on day 0 of the experiment. One group of rats (Gr.P/P) concurrently received placebo capsules, which were substituted with new placebo capsules at the time of middle cerebral artery occlusion (MCAo) on day 14. A second group (Gr.E/E) received $17 \beta$-estradiol capsules on day 0 and new $17 \beta$-estradiol capsules on day 14 . A third group (Gr.E/P) received $17 \beta$ estradiol capsules on day 0 , which were substituted with placebo capsules on day 14 . The last group (Gr.P/E) received placebo capsules on day 0 , which were substituted with $17 \beta$-estradiol capsules on day 14 (Figure 1).

\begin{tabular}{|c|c|c|c|}
\hline & $\begin{array}{l}\text { Day 0: } \\
\text { Ovariectomy }\end{array}$ & $\begin{array}{l}\text { Day 14: } \\
\text { MCAo }\end{array}$ & $\begin{array}{l}\text { Day 17: } \\
\text { Sacrifice }\end{array}$ \\
\hline Gr.P/P & Placebo capsule & \multicolumn{2}{|c|}{ Placebo capsule } \\
\hline Gr.E/E & 17ß-estradiol capsule & \multicolumn{2}{|c|}{ 17ß-estradiol capsule } \\
\hline Gr.E/P & 17ß-estradiol capsule & \multicolumn{2}{|c|}{ Placebo capsule } \\
\hline Gr.P/E & Placebo capsule & \multicolumn{2}{|c|}{ 17ß-estradiol capsule } \\
\hline
\end{tabular}

Figure 1 Study flow-chart. All animals were ovariectomized day 0 of the experiment, and the same time Gr.P/P and Gr.P/E received placebo implants, while Gr.E/E and Gr.E/P received estrogen implants. Day 14 of the experiment, all animals underwent 60 minutes of MCAo. During the anesthesia, blood samples were drawn and the implants were replaced with new ones. Gr.P/P and Gr.E/P received placebo implants, while Gr.E/E and Gr.P/E received estrogen implants. On day 17 of the experiment, all animals were sacrificed, trunk blood collected and brains harvested for analysis. 
The wash-out period was omitted in the current experiment because it has been shown to have no effect on the levels of estrogens induced by the administration regimen [23].

\section{Production of silastic capsules}

The silastic capsules consisted of thirty $\mathrm{mm}$ segments of silastic laboratory tubing (inner/outer diameter: 1.575/ $3.175 \mathrm{~mm}$, Dow Corning, VWR International, Buffalo Grove, IL, USA) filled with sesame oil (placebo) or a solution of $180 \mu \mathrm{g} 17 \beta$-estradiol/mL sesame oil. Five mm pieces of wooden applicator sticks (birch, length: $15 \mathrm{~cm}$, diameter: $2 \mathrm{~mm}$, SelefaTrade AB, Spånga, Sweden) were cut using a fine tooth saw and used to seal the silastic tubing, resulting in an oil-17 $\beta$-estradiol-filled column 20 $\mathrm{mm}$ in length. The capsules were stored overnight in a vial containing sesame oil with the same concentration of $17 \beta$-estradiol as inside the capsules. Before implantation the capsules were carefully wiped. A $0.5 \mathrm{~cm}$ incision was made in the loose skin of the rat's neck, and a pocket was bluntly dissected caudally, in which the silastic capsule was gently installed using forceps. The incision was subsequently closed by a suture. This $17 \beta$-estradiol administration regimen produces hormone serum levels that are within the physiological range and significantly different to ovariectomized controls for at least four weeks (Figure 2) [23].

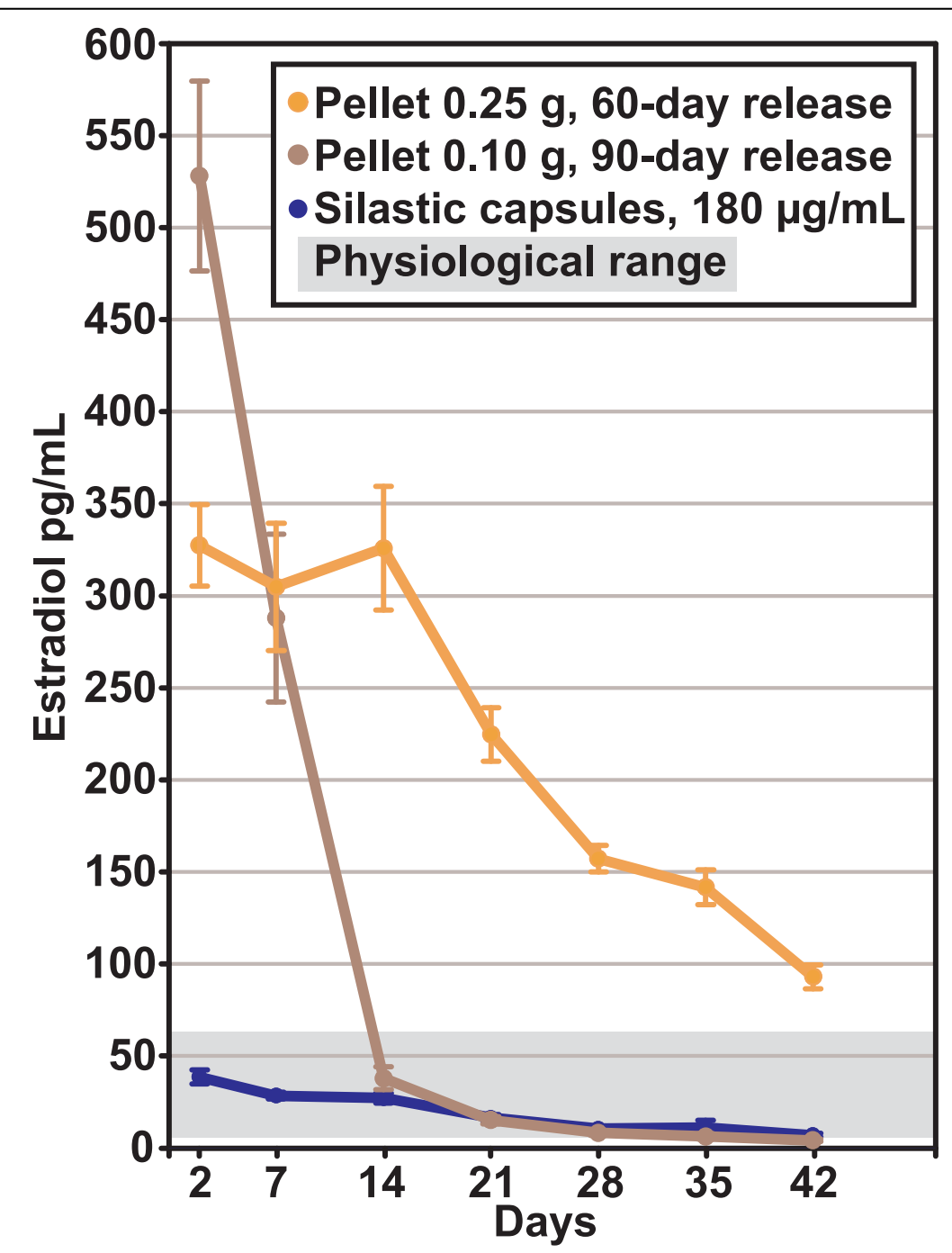

Figure 2 Serum estradiol concentrations depends on administration mode. The three most commonly-used methods for administrating 17/-estradiol to rats were tested in a previous study [23]: slow-release pellets ( $0.25 \mathrm{mg} 60$ day-release pellets, $0.10 \mathrm{mg} 90$ day-release pellets) and silastic capsules (silastic laboratory tubing, inner/outer diameter: 1.575/3.175 mm, filled with $20 \mathrm{~mm}$ columns of $180 \mu \mathrm{\mu g} 173$-estradiol/mL sesame oil). Blood samples were obtained at days $2,7,14,21,28,35$ and 42 after administration, and hormone levels were analyzed. As can be observed in the graph, silastic capsules produced 173-estradiol concentrations that were within the physiological range for at least 4 weeks, while the pellets resulted in early supraphysiological peaks followed by a substantial decrease. 


\section{Middle cerebral artery occlusion}

Fourteen days after ovariectomy and silastic capsule implantation all animals underwent MCAo according to the following procedure. Anesthesia was induced by $4.2 \%$ isoflurane in a mixture $(30 \% / 70 \%)$ of oxygen/nitrous oxide in an induction chamber. A soft endotracheal tube was used for controlled ventilation (Zoovent, CWC600AP, ULV Ltd., UK) using 1.2-1.4\% isoflurane in a mixture (30\%/70\%) of oxygen/nitrous oxide. The tidal volume and ventilation frequency were carefully regulated using on site monitoring of blood gases and acid/base status (AVL, OPTI 1 Medical Nordic AB, Stockholm, Sweden). The rats were placed with their left side up on a thermostatic heating pad (Harvard Homeothermic Blanket System, Edenbridge, UK) to maintain the core/rectum temperature at $37.0 \pm 0.5^{\circ} \mathrm{C}$. The left femoral artery was cannulated using a soft catheter (Micro-renathane ${ }^{\circledast}$ tubing, MRE-025 Braintree Scientific Inc., MA) primed with saline containing heparin (100 IU/mL, Lovens, Malmö, Sweden) for registration of blood pressure (Blood Pressure Transducer, 56360, Stoelting, IL, USA) and pulse (Acq- Knowledge software, BioPac system, Goleta, California, USA). Using an operating microscope (Zeiss Opmi 6-H West Germany), the left MCA was exposed transcranially [25] removing part of the zygomatic bone but keeping the temporal muscle and the facial and mandibular nerves. The MCA was temporarily occluded during 60 min with a microclip between the rhinocortical branch and the lenticulostriate artery [26].

\section{Measuring the size of the ischemic brain lesions}

On day 17 of the experiment, three days after MCAo, the rats were anesthetized by pure carbon dioxide and sacrificed by means of a rat guillotine. The brain was carefully dissected out and cooled in $+4^{\circ} \mathrm{C}$ saline. Two millimeter thick coronary slices of the brain were cut out with razor blades directed by a rat brain matrix (RBM-4000, ASI Instrument Inc., USA) at bregma and 2, 4, 6, $8 \mathrm{~mm}$ posterior and 2 and $4 \mathrm{~mm}$ anterior to bregma. The slices were freed from the dura mater and soaked for $10 \mathrm{~min}$ in a solution of $2 \%$ 2,3,5-triphenyltetrazolium hydrochloride (TTC) in $0.1 \mathrm{~mol} / \mathrm{L}$ PBS (pH 7.4) in a small Petri dish, maintained at $37^{\circ} \mathrm{C}$ in a heater. Gentle stirring of the slices was used to ensure even exposure of the surfaces to staining. Excess TTC was then drained, and the slices were scanned (ScanJet 2c, Hewlett-Packard). The size of brain lesion was measured using SigmaScan Pro version 5 (SPSS Inc.) using an automatic threshold of $40 \%$ in the green spectrum in a similar manner as described by Bederson et al. and Goldlust et al. [27,28].

\section{Blood samples}

Blood samples for $17 \beta$-estradiol analysis was obtained by venipuncture of the hind limb into serum tubes
(Vacuette ${ }^{\circledast}$ Serum Tubes, Hettich Labinstrument AB, Sollentuna, Sweden) during MCAo anesthesia in all animals day 14 of the experiment. Also, five of the animals in Gr. $\mathrm{E} / \mathrm{E}$ and eight of the animals in Gr.E/P had blood samples taken day 16 of the experiment. Only $700 \mu \mathrm{L}$ of blood was withdrawn to minimize animal stress. Day 17 of the experiment, animals were sacrificed and trunk blood collected from all groups for $17 \beta$-estradiol analysis.

\section{$17 \beta$-estradiol radioimmunoassay}

Serum levels of $17 \beta$-estradiol were analyzed using an ${ }^{125} \mathrm{I}$ radioimmunoassay kit (17 $\beta$-estradiol double antibody, KE2D, Siemens Healthcare Diagnostics Inc., Tarrytown, NY, USA), which has previously been tested in rat sera against other radioimmunoassays [29], and measured for 300 seconds in a gamma counter (Gamma Master 1277; Wallac-Pharmacia, Turku, Finland). This method has a lowest detectable concentration of $1.4 \mathrm{pg} / \mathrm{mL}$ and intraand interassay coefficients of variation of $4-13 \%$ and $3.5-$ $5.5 \%$, respectively, depending on the concentration. All samples in the study were analyzed in one single run. Two standard curves were analyzed and all samples were read against their closest standard curve. A volume of $100 \mu \mathrm{L}$ of serum was used for each tube in standard curves and samples. Standards and samples were analyzed in duplicate.

Four samples from a previous experiment including cycling female rats [23] were analyzed in the same batch to allow standardizing factorization of the values.

\section{Protocol violations \\ Excluded animals}

One rat in Gr.E/P was excluded because of technical problems during surgery.

\section{Excluded test results}

All scanned brain slice image from two animals, one each in Gr.P/E and Gr.E/P, and one scanned brain slice image from one animal in Gr.E/E was lost in the analysis process.

Measured concentrations of $17 \beta$-estradiol in two blood samples from Gr.P/E and one blood sample from Gr.E/E were excluded due to technical problems.

\section{Statistics}

The mean and standard error of the mean are used to describe central tendency and variation throughout the article. Size of ischemic brain lesions and $17 \beta$-estradiol concentrations were analyzed with Kruskal-Wallis nonparametric one-way analysis of variance and multiple comparisons [30]. Differences in ischemic lesion sizes between rats with and without the extra sampling occasion in Gr.E/E and Gr.E/P were analyzed by means of Mann-Whitey's test, using the same computer program as above. Differences with $\mathrm{p}$-values $<0.05$ were 
considered significant. The ischemic lesions are presented as percent of the total slice area.

\section{Results}

Impact of 17/-estradiol on size of ischemic brain lesion

The overall mean ischemic lesion of all slices were significantly smaller in Gr.E/E (3.85 $\pm 0.70 \%)$ than in Gr.P/ $\mathrm{P}(7.15 \pm 0.27 \% ; \mathrm{p}=0.015)$. There were no significant differences between Gr.P/P and the two groups Gr.E/P and Gr.P/E, neither in combined mean ischemic lesion area nor in any specific slice. The lesions in Gr.P/E $(10.02 \pm 1.23 \%)$ were significantly larger than in Gr.E/E and Gr.E/P $(5.41 \pm 0.95 \% ; \mathrm{p}<0.001$ and $\mathrm{p}=0.009$ respectively; Figure 3).

The mean lesion sizes were largest in the bregma position $(10.61 \pm 2.49 \%$ to $20.58 \pm 2.61 \%)$ and smallest in bregma $-8 \mathrm{~mm}$ position $(0.0$ to $0.32 \pm 0.30 \%)$ in all groups (Figure 4).

\section{Physiologic parameters}

The blood gases and blood pressures presented in Table 1 were registered immediately prior to the MCAo.

\section{Serum levels of $17 \beta$-estradiol}

On the day for MCAo (day 14) Gr.E/E and Gr.E/P had significantly higher concentrations of serum $17 \beta$-estradiol than had Gr.P/P and Gr.P/E, as expected from the preceding hormone treatments (all p-values $<0.05$ ). Day 17 , the last day of the experiment, the $17 \beta$-estradiol concentrations in Gr.E/E were significantly higher than in Gr.P/P ( $<0.0001)$ and in Gr.E/P ( $\mathrm{p}=0.024)$, and the levels in Gr.P/E were significantly higher than in Gr.P/P $(p=0.0039)$, as expected from the second round of hormone treatments. However, the concentrations in Gr.P/E were not significantly higher than in Gr.E/P. Serum $17 \beta$ estradiol concentrations are presented in Table 2.

\section{Possible effect of venipuncture on the size of the ischemic lesion}

The mean lesion area of the thirteen rats in Gr.E/E and Gr.E/P that underwent an extra venipuncture on day 16 did not differ from the other rats in these groups (4.71 $\pm 0.57 \%$ versus $4.34 \pm 0.50 \% ; \mathrm{p}=0.38$ ).

\section{Discussion}

$17 \beta$-estradiol protects against ischemic brain damage from transient MCAo in female Sprague Dawley rats when administered to provide physiological serum concentrations using silastic capsules both before and after MCAo. No significant neuroprotection was found when the $17 \beta$-estradiol was administered only during the two weeks before or the three days immediately after MCAo.

The main purpose of the current experiment was complementing an earlier study from our lab, using a different method for $17 \beta$-estradiol administration, and administering $17 \beta$-estradiol only before or after the ischemic episode. Silastic capsules were used in the present study instead of slow-release pellets from the

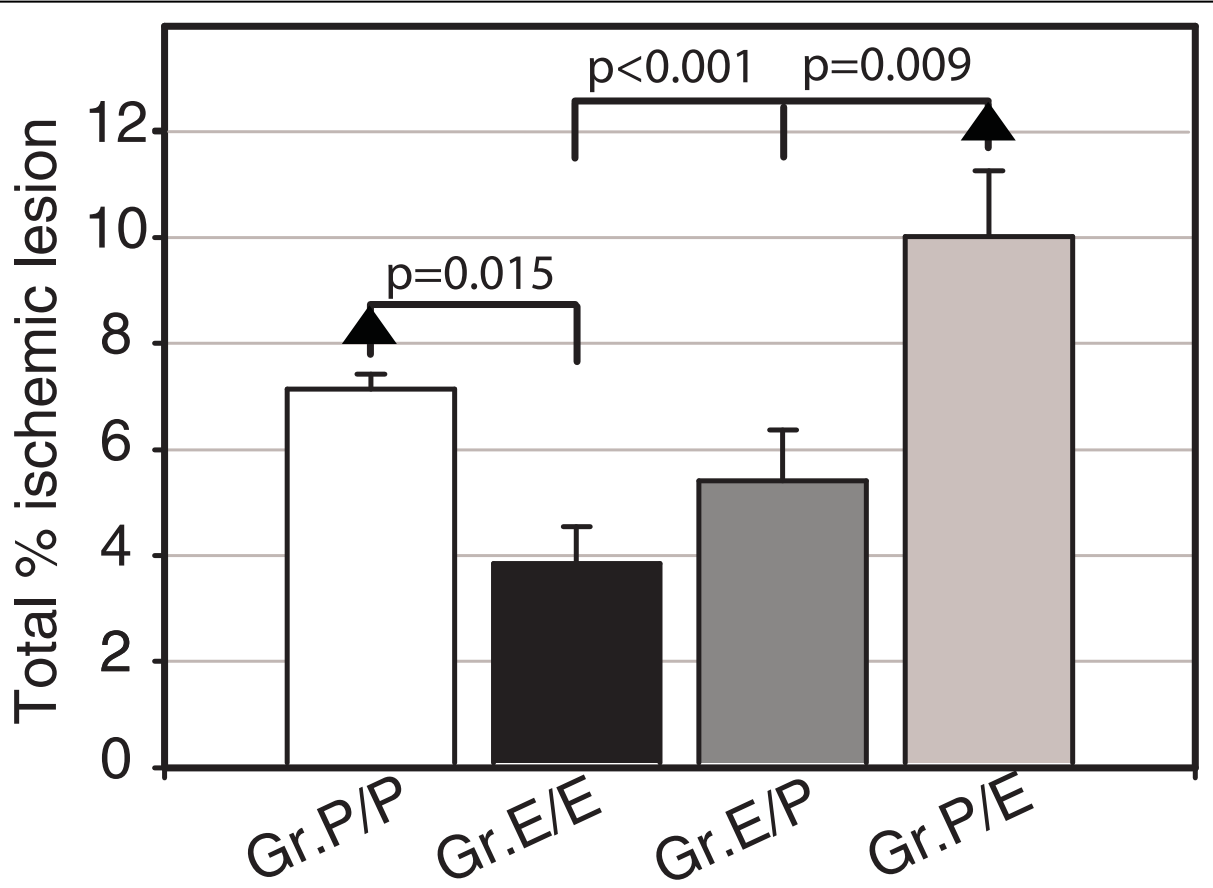

Figure 3 Total ischemic brain lesion. The mean ischemic lesion area calculated from all slices in each experimental group. Gr.P/P had significantly larger lesions than Gr.E/E $(p=0.015)$. 


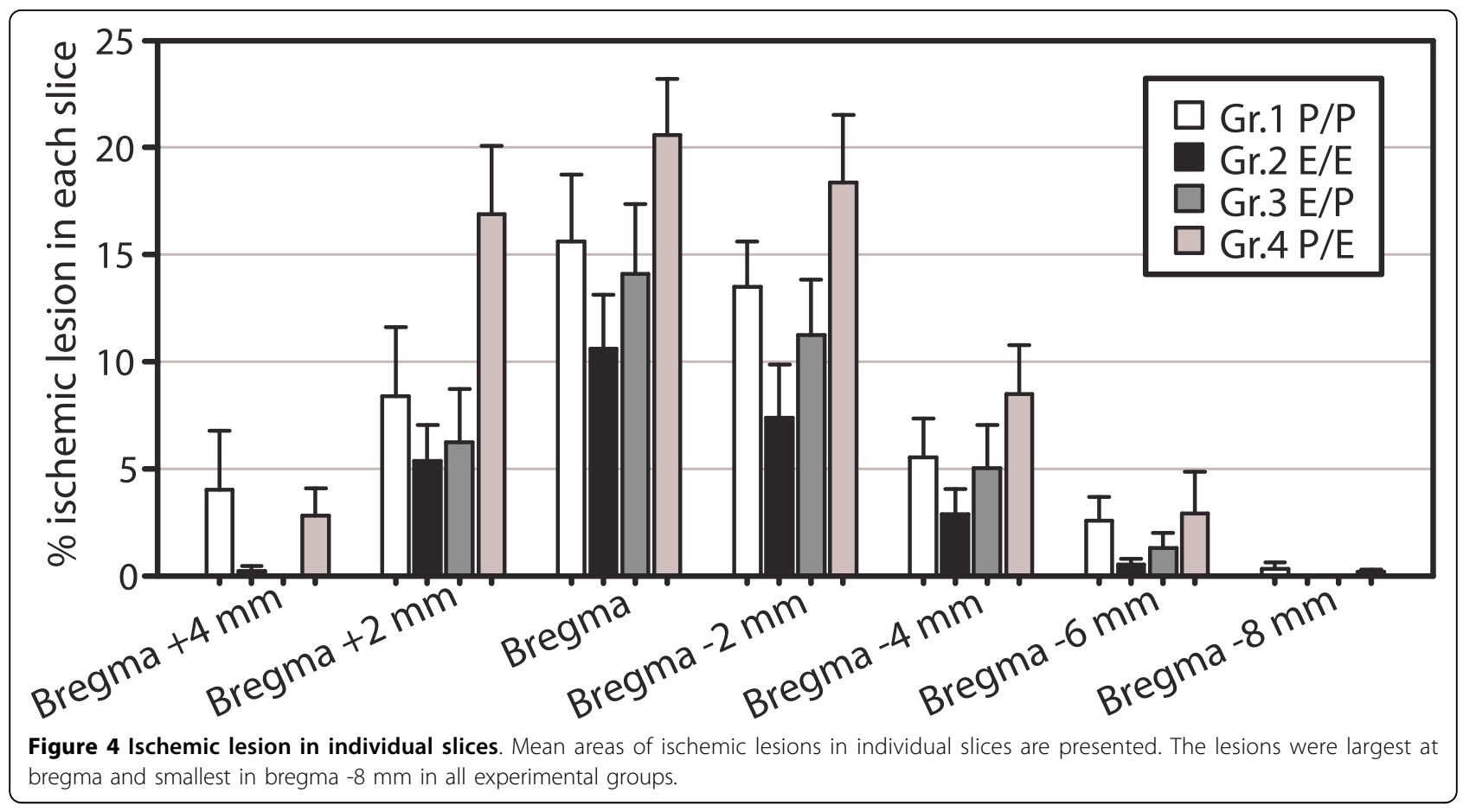

Table 1 Physiological parameters in rats subjected to transient MCA occlusion

\begin{tabular}{llllll}
\hline Group & $\mathbf{n}$ & $\mathbf{p 0}_{\mathbf{2}}(\mathbf{k P a})$ & $\mathbf{p C O} \mathbf{2}_{\mathbf{2}} \mathbf{( k P a )}$ & $\mathbf{p H}$ & $\begin{array}{l}\text { Systolic blood } \\
\text { pressure }(\mathbf{m m} \mathbf{H g})\end{array}$ \\
\hline Gr.P/P & 12 & $12.1 \pm 0.9$ & $5.1 \pm 0.3$ & $7.52 \pm 0.04$ & $100 \pm 4$ \\
\hline Gr.E/E & 12 & $14.7 \pm 1.3$ & $5.2 \pm 0.2$ & $7.49 \pm 0.01$ & $113 \pm 3$ \\
\hline Gr.E/P & 11 & $16.1 \pm 1.3$ & $5.0 \pm 0.1$ & $7.48 \pm 0.01$ & $110 \pm 3$ \\
\hline Gr.P/E & 12 & $12.8 \pm 2.4$ & $5.5 \pm 0.4$ & $7.49 \pm 0.03$ & $105 \pm 9$ \\
\hline
\end{tabular}

Table 2 Concentrations of $17 \beta$-estradiol (mean \pm SEM pg/mL)

\begin{tabular}{llll}
\hline Group & Day $\mathbf{1 4}$ (MCAo) & Day $\mathbf{1 6}$ & Day $\mathbf{1 7}$ (sacrifice) \\
\hline Gr.P/P & $1.5 \pm 0.7$ & Not measured & $2.5 \pm 1.8$ \\
\hline Gr.E/E & $10.1 \pm 5.4$ & $35.6 \pm 8.5$ & $32.4 \pm 8.5$ \\
\hline Gr.E/P & $12.8 \pm 4.7$ & $8.0 \pm 4.6$ & $7.7 \pm 2.1$ \\
\hline Gr.P/E & $1.6 \pm 1.0$ & Not measured & $11.8 \pm 3.5$ \\
\hline
\end{tabular}

company IRA in the previous study (Table 3) [21]. Since neuroprotection of $17 \beta$-estradiol was found in the current study, while the opposite was found in the previous experiment, the choice of administration method seems to have a crucial effect on the results. Importantly, this effect is not confined to producing different degrees of neuroprotection, but the slow-release pellets actually cause neurodamage instead of neuroprotection. This is in concordance with a previous review comparing methods of studies reporting neuroprotection versus neurodamage of estrogen, showing that $17 \beta$-estradiol has only been reported to be neurodamaging when slow-release pellets from IRA have been used [22].

The reasons for discrepancies in results from the silastic capsule and pellet method of administration of $17 \beta$-estradiol are probably the markedly different serum concentration profiles produced (Figure 2). In previous experiments in our lab we have shown that the pellets result in extreme, supraphysiological levels for several days or weeks, followed by a substantial decrease, while silastic capsules produces serum levels that are, although steadily decreasing, within the physiological range for at least four weeks $[23,31]$. In the light of these administration method studies it seems reasonable to conclude that the early, prolonged, supraphysiological peak of the pellets in the context of cerebral ischemia exerts detrimental effects on the brain.

The time aspect of estrogens' effects on cerebral ischemia, investigated in the current study, has been addressed in a few previous studies. In 1997, Simpkins et al. compared infarct volumes when $17 \beta$-estradiol had been intravenously administered $24 \mathrm{~h}$ before, $40 \mathrm{~min}$ and 90 min after ischemic damage respectively and found a protective effect at all three time points [10]. In 2000, Yang et al. administered $17 \beta$-estradiol through intravenous injections and silastic capsules (filled with crystalloid hormone, differing from the oil-solution adopted in the current experiment) at 0.5, 1, 2, 3 and 4 hours after onset of ischemia finding less neuroprotection with later administration [32]. Saleh et al. conducted two experiments in 2001 where the time aspect 
Table 3 Methodological comparison of previous and present study

\begin{tabular}{lcc}
\hline Methodological parameter & Theodorsson \& Theodorsson 2005 & Present study \\
\hline Estrogen administration mode & Pellets from Innovative Research of America & Home-made silastic capsules \\
\hline Length of estrogen treatment & 2 weeks \\
\hline Type of estrogen & MCAo by microclip, reperfusion after 60 min \\
\hline Method for induction of ischemic lesions & 72 hours \\
\hline Length of time between ischemia and damage evaluation & TTC-staining and infarct size measurement \\
\hline Type of outcome measure & Female \\
\hline Rat sex & Sprague-Dawley \\
\hline Rat strain & 3 months \\
\hline Rat age & None \\
\hline Rat diseases & Same laboratory, surgeon and technical equipment used in both studies \\
\hline Miscellaneous & Neurodamage $(\mathrm{p}<0.001)$ \\
\hline Effect of $17 \beta$-estradiol on cerebral ischemia & Neuroprotection ( $\mathrm{p}=0.015)$ \\
\hline
\end{tabular}

of estrogens effect on cerebral ischemia was investigated. In one, subcutaneous injections of $17 \beta$-estradiol were administered at $30 \mathrm{~min}$ before ischemia, at the onset of ischemia and at $30 \mathrm{~min}$ after the onset of ischemia respectively. It was found that administration $30 \mathrm{~min}$ before ischemia produced the most substantial neuroprotection, and that administration $30 \mathrm{~min}$ after ischemia did not significantly influence the infarct size [12]. In the other, the hormone was administered by intraventricular infusion at 30 and $10 \mathrm{~min}$ before ischemia respectively, with the result that more protection was obtained from the earlier time point [33]. In 2007, Liu et al. administered subcutaneous injections of $17 \beta$-estradiol at $30 \mathrm{~min}$ and at 6 hours after onset of ischemia respectively, only finding neuroprotection from the first time of injection if all rats were counted [34]. The narrow time span for hormone administration, differing at most $25.5 \mathrm{~h} \mathrm{[10],} \mathrm{and} \mathrm{the} \mathrm{administration} \mathrm{methods}$ adopted in all of these previous studies contrast strongly with the current study, which makes precise comparisons difficult. However, our present observations suggest that administering estrogens by means of slow-release silastic tubes at the time of ischemia lacks the protective properties of a long-term estrogen treatment, which is in contrasts to earlier results $[10,32,34]$. Thus, the current results indicate that administering $17 \beta$-estradiol only at the time of stroke by silastic capsules is ineffective, and that the protective potential of $17 \beta$-estradiol is facilitated by prophylactic long-term administration. However, the impact of estrogens during the development of the ischemic damage is suggested by the fact that no significant protection was observed in Gr.E/P, receiving only estrogen pretreatment, while protection is seen when at-stroke estrogens are added to this regime, as in Gr.E/E.

Similar reasoning can be applied when considering the results from Gr.E/P. Although this regime seems to lack protective properties by itself, the partial neuroprotective of estrogen pretreatment is suggested by the lack of protection in Gr.P/E in comparison to Gr.E/E. To our knowledge, no study investigating the effect of $17 \beta$ estradiol pretreatment, interrupted at the time of ischemic damage, has been previously reported.

The $17 \beta$-estradiol concentrations in Gr.E/P and Gr.P/E did not differ significantly on the last day of the experiment. Because the half-life of $17 \beta$-estradiol in the blood is measured in minutes, the comparatively small decrease in hormone levels in Gr.E/P was unexpected. A possible reason could be that although the hormone-containing capsules had been replaced with placebo capsules, some of the hormone could still have been left in the subcutis. This hypothesis is supported by the lack of hormone level decrease between days 14 to 17 in Gr.E/P.

\section{Possible mechanisms}

The elucidation of the mechanisms behind estrogens effects on cerebral ischemia is of fundamental importance for the possibility of directly affecting the pathological process. Many mechanisms have been suggested to be involved in the protective effects of $17 \beta$-estradiol in the brain, being mediated by intracellular receptors, membrane receptors and by direct chemical interactions including antioxidant effects [35]. Interestingly, the antioxidant effects of estrogens (phenolic scavenging of lipid peroxyl radicals) may prevail at physiological concentrations whereas redox-cycling at higher estrogen concentrations may lead to increased radical generation and damage [36,37]. Estrogens may also protect against neurodamage by antithrombogenic actions through effects on smooth vascular muscle and endothelium, systemic effects reducing total cholesterol and LDL concentrations, and through effects on hepatic expression of genes for several coagulation and fibrinolytic proteins. Also, estrogens have direct effects on neurons by 
increasing transcription of antiapoptotic genes, decreasing transcription of apoptotic genes, reducing mitochondrial release of Cytochrome $\mathrm{C}$, interacting with neurotrophins, and have anti-inflammatory effects through attenuation of TNF- $\alpha$ (reviewed in $[38,39]$ ). Damaging effects on the nervous system by estrogens have been suggested to result from the formation of free radicals [40] or from increased excitotoxicity [41] by upregulating glutamate NMDA receptors [42], inducing seizure activity [43] and decreasing glutamate uptake by astrocytes [44]. Thus, it seems reasonable that all these mechanisms, differing in both route of exertion and individual dose-response relations, together could create a highly complex, e.g. biphasic, dose-response relation.

\section{Conclusions}

The hypothesis that the mode of estrogen administration dictates the hormone's effect on ischemic damage by producing specific hormone release profiles is corroborated by the present study. It is important to realize that this is not merely a methodological question concerning rat models, but suggests a fundamental property of estrogens' actions in the nervous system. Thus, it does not only have relevance for the design of future animal studies; it also has a bearing on the debate on peri-menopausal hormone replacement therapy. Perhaps altered administration regimens resulting in lower plasma concentrations may reduce the hazardous effects and even have favorable effects, while preserving the relief of peri-menopausal symptoms?

\footnotetext{
Abbreviations

Gr.E/E: Experimental group receiving $17 \beta$-estradiol capsules both before and after MCAo; Gr.E/P: Experimental group receiving17 $\beta$-estradiol capsules before and placebo capsules after MCAo; Gr.P/E: Experimental group receiving placebo capsules before and $17 \beta$-estradiol capsules after MCAo; Gr. P/P: Experimental group receiving placebo capsules both before and after MCAo; IRA: Innovative Research of America; MCA: Middle cerebral artery; MCAo: Middle cerebral artery occlusion; TTC: 2,3,5-triphenyltetrazolium hydrochloride.
}

\section{Acknowledgements}

Funding: The County Council of Östergötland, Sweden.

\section{Author details}

${ }^{1}$ Institution of Clinical and Experimental Medicine/Department of Clinical Chemistry, Linkoping University, Linkoping, Sweden. ${ }^{2}$ Institution of Clinical and Experimental Medicine/Department of Neurosurgery, Linkoping University, Linkoping, Sweden.

\section{Authors' contributions}

JOS contributed by participating in planning/designing the study, producing the $17 \beta$-estradiol capsules, performing the hormone assays, assessing the data, and by drafting the article. ET contributed by participating in planning/ designing the study, assessing the data and by revising the manuscript. LH contributed by performing all surgical procedures, obtaining all necessary samples, analyzing infarct sizes and by revising the manuscript. AT contributed by participating in planning/designing the study, analyzing infarct sizes, assessing the data and by revising the manuscript. All authors read and approved the final manuscript.

\section{Competing interests}

The authors declare that they have no competing interests.

Received: 5 September 2009 Accepted: 17 March 2010

Published: 17 March 2010

\section{References}

1. Stegmayr B, Asplund K, Kuulasmaa K, Rajakangas AM, Thorvaldsen P Tuomilehto J: Stroke incidence and mortality correlated to stroke risk factors in the WHO MONICA Project. An ecological study of 18 populations. Stroke 1997, 28(7):1367-1374.

2. Sacco RL, Boden-Albala B, Gan R, Chen X, Kargman DE, Shea S, Paik MC Hauser WA: Stroke incidence among white, black, and Hispanic residents of an urban community: the Northern Manhattan Stroke Study. Am J Epidemiol 1998, 147(3):259-268.

3. Kolominsky-Rabas PL, Weber M, Gefeller O, Neundoerfer B, Heuschmann PU: Epidemiology of ischemic stroke subtypes according to TOAST criteria: incidence, recurrence, and long-term survival in ischemic stroke subtypes: a population-based study. Stroke 2001, 32(12):2735-2740.

4. Falkeborn M, Persson I, Terent A, Adami HO, Lithell $H$, Bergstrom R: Hormone replacement therapy and the risk of stroke. Follow-up of a population-based cohort in Sweden. Arch Intern Med 1993, 153(10):1201-1209.

5. Paganini-Hill A, Ross RK, Henderson BE: Postmenopausal oestrogen treatment and stroke: a prospective study. Bmj 1988, 297(6647):519-522.

6. Hulley S, Grady D, Bush T, Furberg C, Herrington D, Riggs B, Vittinghoff E: Randomized trial of estrogen plus progestin for secondary prevention of coronary heart disease in postmenopausal women. Heart and Estrogen/ progestin Replacement Study (HERS) Research Group. Jama 1998, 280(7):605-613.

7. Simon JA, Hsia J, Cauley JA, Richards C, Harris F, Fong J, Barrett-Connor E, Hulley SB: Postmenopausal hormone therapy and risk of stroke: The Heart and Estrogen-progestin Replacement Study (HERS). Circulation 2001, 103(5):638-642

8. Viscoli CM, Brass LM, Kernan WN, Sarrel PM, Suissa S, Horwitz RI: A clinical trial of estrogen-replacement therapy after ischemic stroke. N Engl J Med 2001, 345(17):1243-1249.

9. Rossouw JE, Anderson GL, Prentice RL, LaCroix AZ, Kooperberg C, Stefanick ML, Jackson RD, Beresford SA, Howard BV, Johnson KC, et al: Risks and benefits of estrogen plus progestin in healthy postmenopausal women: principal results From the Women's Health Initiative randomized controlled trial. Jama 2002, 288(3):321-333.

10. Simpkins JW, Rajakumar G, Zhang YQ, Simpkins CE, Greenwald D, Yu CJ Bodor N, Day AL: Estrogens may reduce mortality and ischemic damage caused by middle cerebral artery occlusion in the female rat. J Neurosurg 1997, 87(5):724-730.

11. Dubal DB, Wise PM: Neuroprotective effects of estradiol in middle-aged female rats. Endocrinology 2001, 142(1):43-48.

12. Saleh $T M, C r i b b ~ A E$, Connell BJ: Estrogen-induced recovery of autonomic function after middle cerebral artery occlusion in male rats. Am J Physiol Regul Integr Comp Physiol 2001, 281(5):R1531-1539.

13. McCullough LD, Alkayed NJ, Traystman RJ, Williams MJ, Hurn PD: Postischemic estrogen reduces hypoperfusion and secondary ischemia after experimental stroke. Stroke 2001, 32(3):796-802.

14. Koh PO, Cho JH, Won CK, Lee HJ, Sung JH, Kim MO: Estradiol attenuates the focal cerebral ischemic injury through mTOR/p70S6 kinase signaling pathway. Neurosci Lett 2008, 436(1):62-66.

15. Zhang YQ, Shi J, Rajakumar G, Day AL, Simpkins JW: Effects of gender and estradiol treatment on focal brain ischemia. Brain Res 1998, 784(12):321-324.

16. Gordon KB, Macrae IM, Carswell HV: Effects of 17beta-oestradiol on cerebral ischaemic damage and lipid peroxidation. Brain Res 2005 1036(1-2):155-162.

17. Harukuni I, Hurn PD, Crain BJ: Deleterious effect of beta-estradiol in a rat model of transient forebrain ischemia. Brain Res 2001, 900(1):137-142.

18. Yong Y, Xie HJ, Zhang YF, Yang QD, Liao DF, Yang HL, Yan PK, Liu ZJ: 17beta-estradiol potentiates ischemia-reperfusion injury in diabetic ovariectomized female rats. Brain Res 2005, 1054(2):192-199.

19. Carswell HV, Bingham D, Wallace K, Nilsen M, Graham DI, Dominiczak AF, Macrae IM: Differential effects of 17beta-estradiol upon stroke damage in 
stroke prone and normotensive rats. J Cereb Blood Flow Metab 2004, 24(3):298-304.

20. Bingham D, Macrae IM, Carswell HV: Detrimental effects of 17betaoestradiol after permanent middle cerebral artery occlusion. J Cereb Blood Flow Metab 2005, 25(3):414-420.

21. Theodorsson A, Theodorsson E: Estradiol increases brain lesions in the cortex and lateral striatum after transient occlusion of the middle cerebral artery in rats: no effect of ischemia on galanin in the stroke area but decreased levels in the hippocampus. Peptides 2005, 26(11):2257-2264.

22. Strom JO, Theodorsson A, Theodorsson E: Dose-related neuroprotective versus neurodamaging effects of estrogens in rat cerebral ischemia: a systematic analysis. J Cereb Blood Flow Metab 2009, 29(8):1359-1372.

23. Strom JO, Theodorsson E, Theodorsson A: Order of magnitude differences between methods for maintaining physiological 17beta-oestradiol concentrations in ovariectomized rats. Scand J Clin Lab Invest 2008, 68(8):814-822.

24. Soderstrom I, Strand M, Ingridsson AC, Nasic S, Olsson T: 17beta-estradiol and enriched environment accelerate cognitive recovery after focal brain ischemia. Eur J Neurosci 2009, 29(6):1215-1224.

25. Tamura A, Graham DI, McCulloch J, Teasdale GM: Focal cerebral ischaemia in the rat: 1 . Description of technique and early neuropathological consequences following middle cerebral artery occlusion. J Cereb Blood Flow Metab 1981, 1(1):53-60.

26. Theodorsson A, Holm L, Theodorsson E: Modern anesthesia and peroperative monitoring methods reduce per- and postoperative mortality during transient occlusion of the middle cerebral artery in rats. Brain Res Brain Res Protoc 2005, 14(3):181-190.

27. Bederson JB, Pitts LH, Germano SM, Nishimura MC, Davis RL, Bartkowski HM Evaluation of 2,3,5-triphenyltetrazolium chloride as a stain for detection and quantification of experimental cerebral infarction in rats. Stroke 1986, 17(6):1304-1308

28. Goldlust EJ, Paczynski RP, He YY, Hsu CY, Goldberg MP: Automated measurement of infarct size with scanned images of triphenyltetrazolium chloride-stained rat brains. Stroke 1996, 27(9):1657-1662.

29. Strom JO, Theodorsson A, Theodorsson E: Substantial discrepancies in 17beta-oestradiol concentrations obtained with three different commercial direct radioimmunoassay kits in rat sera. Scand J Clin Lab Invest 2008, 68(8):806-813.

30. Theodorsson-Norheim E: Kruskal-Wallis test: BASIC computer program to perform nonparametric one-way analysis of variance and multiple comparisons on ranks of several independent samples. Comput Methods Programs Biomed 1986, 23(1):57-62.

31. Theodorsson A, Hilke S, Rugarn O, Linghammar D, Theodorsson E: Serum concentrations of 17 beta-estradiol in ovariectomized rats during two times six weeks crossover treatment by daily injections in comparison with slow-release pellets. Scand I Clin Lab Invest 2005, 65(8):699-705.

32. Yang SH, Shi J, Day AL, Simpkins JW: Estradiol exerts neuroprotective effects when administered after ischemic insult. Stroke 2000, 31(3):745-749, discussion 749-750.

33. Saleh TM, Cribb AE, Connell BJ: Reduction in infarct size by local estrogen does not prevent autonomic dysfunction after stroke. Am J Physiol Regul Integr Comp Physiol 2001, 281(6):R2088-2095.

34. Liu R, Wang X, Liu Q, Yang SH, Simpkins JW: Dose dependence and therapeutic window for the neuroprotective effects of 17beta-estradiol when administered after cerebral ischemia. Neurosci Lett 2007, 415(3):237-241.

35. Behl C: Oestrogen as a neuroprotective hormone. Nat Rev Neurosci 2002 3(6):433-442.

36. Simpkins JW, Wang J, Wang X, Perez E, Prokai L, Dykens JA: Mitochondria play a central role in estrogen-induced neuroprotection. Curr Drug Targets CNS Neurol Disord 2005, 4(1):69-83.

37. Prokai L, Prokai-Tatrai K, Perjesi P, Zharikova AD, Perez EJ, Liu R, Simpkins JW: Quinol-based cyclic antioxidant mechanism in estrogen neuroprotection. Proc Natl Acad Sci USA 2003, 100(20):11741-11746.

38. Alonso de Lecinana M, Egido JA: Estrogens as neuroprotectants against ischemic stroke. Cerebrovasc Dis 2006, 21(Suppl 2):48-53.

39. Amantea D, Russo R, Bagetta G, Corasaniti MT: From clinical evidence to molecular mechanisms underlying neuroprotection afforded by estrogens. Pharmacol Res 2005, 52(2):119-132.
40. Desjardins GC, Beaudet A, Meaney MJ, Brawer JR: Estrogen-induced hypothalamic beta-endorphin neuron loss: a possible model of hypothalamic aging. Exp Gerontol 1995, 30(3-4):253-267.

41. Macrae IM, Carswell HV: Oestrogen and stroke: the potential for harm as well as benefit. Biochem Soc Trans 2006, 34(Pt 6):1362-1365.

42. Weiland NG: Glutamic acid decarboxylase messenger ribonucleic acid is regulated by estradiol and progesterone in the hippocampus. Endocrinology 1992, 131(6):2697-2702.

43. Foy MR, Xu J, Xie X, Brinton RD, Thompson RF, Berger TW: 17beta-estradiol enhances NMDA receptor-mediated EPSPs and long-term potentiation. $J$ Neurophysiol 1999, 81(2):925-929.

44. Sato K, Matsuki N, Ohno Y, Nakazawa K: Estrogens inhibit I-glutamate uptake activity of astrocytes via membrane estrogen receptor alpha. J Neurochem 2003, 86(6):1498-1505.

\section{doi:10.1186/1471-2202-11-39}

Cite this article as: Strom et al:: Different methods for administering $17 \beta$-estradiol to ovariectomized rats result in opposite effects on ischemic brain damage. BMC Neuroscience 2010 11:39.

\section{Submit your next manuscript to BioMed Central and take full advantage of:}

- Convenient online submission

- Thorough peer review

- No space constraints or color figure charges

- Immediate publication on acceptance

- Inclusion in PubMed, CAS, Scopus and Google Scholar

- Research which is freely available for redistribution
C Biomed Central 